\title{
Intruder Activity Analysis Under Unreliable Sensor Networks
}

\author{
IEEE Conference on Automation \\ Science and Engineering
}

\author{
Tae-Sic Yoo \\ Humberto E. Garcia
}

U.S. Department of Energy

National Laboratory

operated by

Battelle Energy Alliance

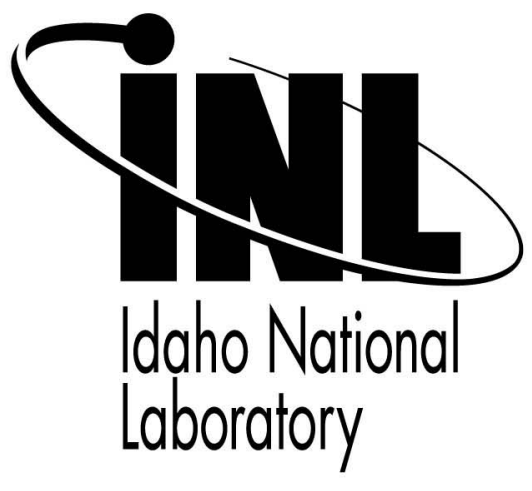

This is a preprint of a paper intended for publication in a journal or proceedings. Since changes may be made before publication, this preprint should not be cited or reproduced without permission of the author. This document was prepared as an account of work sponsored by an agency of the United States Government. Neither the United States Government nor any agency thereof, or any of their employees, makes any warranty, expressed or implied, or assumes any legal liability or responsibility for any third party's use, or the results of such use, of any information, apparatus, product or process disclosed in this report, or represents that its use by such third party would not infringe privately owned rights. The views expressed in this paper are not necessarily those of the United States Government or the sponsoring agency. 


\title{
Intruder Activity Analysis under Unreliable Sensor Networks
}

\author{
Tae-Sic Yoo and Humberto E. Garcia \\ Sensor and Decision Systems Group \\ Idaho National Laboratory, U.S.A. \\ \{Tae-Sic.Yoo, Humberto.Garcia\}@inl.gov
}

\begin{abstract}
This paper addresses the problem of counting intruder activities within a monitored domain by a sensor network. The deployed sensors are unreliable. We characterize imperfect sensors with misdetection and false-alarm probabilities. We model intruder activities with Markov Chains. A set of Hidden Markov Models (HMM) models the imperfect sensors and intruder activities to be monitored. A novel sequential change detection/isolation algorithm is developed to detect and isolate a change from an HMM representing no intruder activity to another HMM representing some intruder activities. Procedures for estimating the entry time and the trace of intruder activities are developed. A domain monitoring example is given to illustrate the presented concepts and computational procedures.
\end{abstract}

\section{INTRODUCTION}

A sensor network consists of spatially distributed sensors and associated signal processors. The design purpose of a sensor network is to detect events of interest and estimate parameters that characterize these events. Sensor network solutions are under active development $[1,3-5,8,11,12,19$, $22,23]$ as the associated deliverables are playing important roles in various application domains. Domain monitoring for intrusion detection is a typical application of sensor networks. In this application, sensors are deployed over a region where intruder activities are to be monitored. For instance, sensor nodes may be deployed over battlefields to detect enemy activities. When the sensors detect an activity event, the event needs to be reported to one of the base stations in order to conduct further information processing or issue appropriate actions.

The base stations receive sequence of reports/observations from spatially distributed sensor devices. In practice, sensor devices are not perfect; e.g., sensors may report false alarms or misdetect activities. The sequence of observations may be generated from real activities to be monitored or may include false alarms from sensing devices. These sensor information should be fused systematically to address the following issues.

Q1: How many intruder activities are occurring within the monitored domain?

Q2: How did intruder activities progress?

Both issues are important in assessing damages, mitigating risk, and allocating resources to cope with intruder activities. Figure 1 depicts the situation to be addressed in this paper.

We find that many related intruder activity detection problems and solutions are available from diverse disciplines and application domains. Relevant works are listed below.

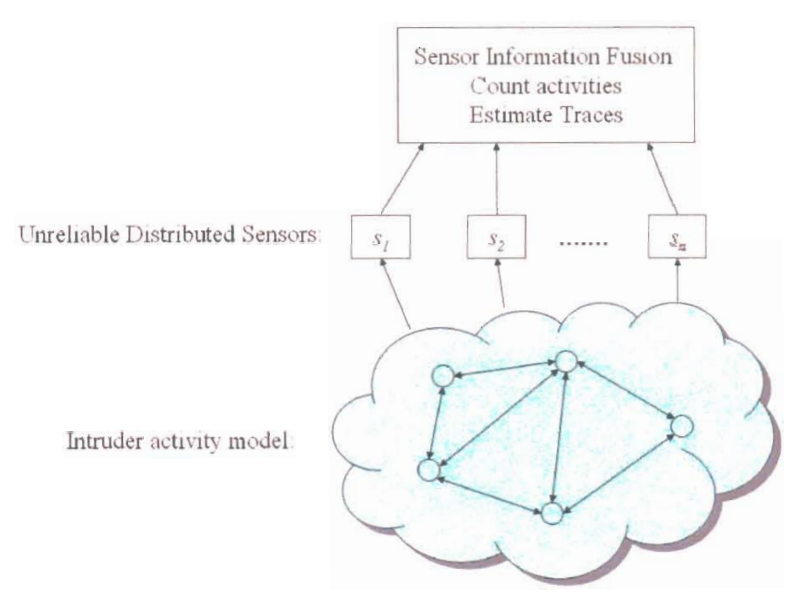

Fig. 1. Conceptual Diagram of Intruder Activity Analysis Architecture

- Victor Klee introduced the art gallery problem in 1973 in a discussion with Vasek Chvatal. The art gallery problem and its variations $[7,9,14,18]$ deal with setting a minimal number of vertex guards in a gallery hall of a complex polygonal shape to secure the visibility of every point in the hall. The objective of these approaches is to secure deterministic visual coverage on areas of interest.

- The objective of intrusion-detection expert systems in the context of [6] and its variations is to detect break-ins, penetrations, and other forms of computer abuse. These approaches mainly rely on building pattern models and designing detection logics.

- Concepts of coverage and exposure in wireless sensor networks $[11,12]$ are measures of surveillance quality provided by a given sensor network. In a similar framework, [4] considers strategies of randomly throwing a batch of sensors to a monitored field in order to achieve a certain intruder exposure level.

- In [5], the authors present algorithms to control and coordinate mobile sensor networks. Unlike art gallery type optimization problems, with controlled mobility, sensors can be located anywhere in a given polygon environment. The main issue is to locate the mobile sensors in order to give optimal coverage.

The remainder of this paper is organized as follows. In Section II, we model multiple intruder activities with 
imperfect sensors via Hidden Markov Models. Section III describes a procedure for counting intruder activities with a sequential detection and isolation procedure. The matrixCUSUM procedure [15] developed for i.i.d. case is modified in order to accommodate sequential observation dependency of HMMs. Section IV addresses the issues of estimating the current intruder activity locations and the trace of intruder activities. In Section V, we apply the developed methodology to monitor an illustrative complex network. Section VI concludes the paper with some remarks.

\section{Modeling Intruder Activities And Unreliable SENSORS WITH HIDDEN MARKOV MODELS}

We use a set of HMMs to model the intruder activities that need to be monitored along with the deployed unreliable sensors. Let us first describe a Markov Chain model for intruder activity.

\section{A. Markov Chain Intruder Activity Model}

We model the network of concern as a strongly connected directed graph $G=(V, E)$ where

$$
V=\left\{v_{1}, v_{2}, \ldots, v_{m}\right\} \text { and } E \subseteq V \times V .
$$

The objective of an intruder is to infiltrate and travel the network and conduct some activities of negative consequences within the network. At regularly-spaced discrete times, the intruder makes transition (possibly back to the same state) according to a set of probabilities associated with the current state. The actual location of the intruder at time $t$ is denoted by $X_{t}$. In this paper, we assume a firstorder Markov stochastic traveling strategy for the intruder in the sense that the next vertex to reach only depends on the current vertex where the intruder resides. That is, $\forall t \in \mathbb{N}$,

$$
\begin{aligned}
& p\left(X_{t+1}=v_{i} \mid X_{t}=v_{j}, X_{t-1}=v_{k}, \ldots\right) \\
= & p\left(X_{t+1}=v_{i} \mid X_{t}=v_{j}\right) .
\end{aligned}
$$

Further we assume that the transition probability is stationary. That is, $\forall t, t^{\prime} \in \mathbb{N}$,

$$
p\left(X_{t+1}=v_{i} \mid X_{t}=v_{j}\right)=p\left(X_{t^{\prime}+1}=v_{i} \mid X_{t^{\prime}}=v_{j}\right) .
$$

We denote the state transition probability distribution by $A=$ $\left\{a_{v_{i}, v_{j}}\right\}$ where

$$
a_{v_{i}, v_{j}}:=p\left(X_{t+1}=v_{i} \mid X_{t}=v_{j}\right), i, j \in\{1, \ldots, m\} .
$$

We denote the initial state probability distribution by

$$
\pi_{1}=\left\{\pi_{1}\left(v_{i}\right)\right\}_{i=1}^{m}
$$

where $\pi_{1}\left(v_{i}\right)=p\left(X_{1}=v_{i}\right)$, for $i \in\{1, \ldots, m\}$.

\section{B. Sensor Model}

We assume that sensors may reside at the set of vertexes $V_{s}=\left\{v_{s_{1}}, v_{s_{2}}, \ldots, v_{s_{n}}\right\} \subseteq V$, with $n$ being the number of deployed sensors that may generate detection events. ${ }^{1}$ As we consider unreliable sensors, we associate each sensor

\footnotetext{
${ }^{1}$ We do not consider the issue of optimizing sensor deployment in this paper. Related efforts regarding the optimization of sensor deployment can be found in [22].
}

with detection and false-alarm probabilities. The detection decision at time $t$ of a sensor located at vertex $v_{s_{i}} \in V_{s}$ is denoted by $d\left(v_{s_{i}}, t\right) \in\{0,1\}$. The detection decision associated with each sensor is assumed to be mutually independent. The associated detection and false alarm probabilities are respectively given $b^{2}$ :

$$
\begin{aligned}
& p_{d}\left(v_{s_{i}}\right):=p\left(d\left(v_{s_{i}}, t\right)=1 \mid X_{t}=v_{s_{i}}\right), \\
& p_{f}\left(v_{s_{i}}\right):=p\left(d\left(v_{s_{i}}, t\right)=1 \mid X_{t} \neq v_{s_{i}}\right) .
\end{aligned}
$$

Figure 2 shows an example of graph and sensor models with $V_{s}=\{2,3\}$.

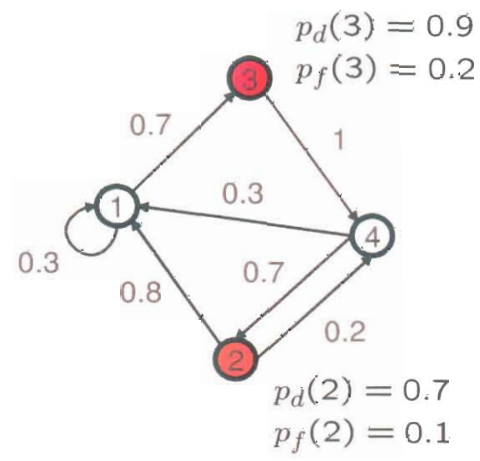

Fig. 2. Intruder activity model with imperfect sensors

Let $D\left(V_{s}, t\right)=\left(d\left(v_{s_{1}}, t\right), \ldots, d\left(v_{s_{n}}, t\right)\right)$ and $\vec{d}:=$ $\left(d_{s_{1}}, d_{s_{2}}, \ldots, d_{s_{n}}\right) \in\{0,1\}^{n}$. We denote the observation symbol probability distribution by $B=\left\{b_{v_{j}}(\vec{d})\right\}$ where, for $v_{j} \in V_{s}$,

$$
\begin{aligned}
b_{v_{j}}(\vec{d}):= & p\left(D\left(V_{s}, \imath\right)=\vec{d} \mid X_{t}=v_{j}\right) \\
= & \prod_{k=1}^{n} p\left(d\left(v_{s_{k}}, t\right)=d_{s_{k}} \mid X_{t}=v_{j}\right) \\
= & p_{d}\left(v_{j}\right)^{d_{j}}\left(1-p_{d}\left(v_{j}\right)\right)^{1-d_{j}} \\
& \cdot \prod_{v_{s_{k}} \in V_{s}, s_{k} \neq j} p_{f}\left(v_{s_{k}}\right)^{d_{s_{k}}}\left(1-p_{f}\left(v_{s_{k}}\right)\right)^{1-d_{s_{k}}},
\end{aligned}
$$

and, for $v_{j} \notin V_{s}$,

$$
b_{v_{j}}(\vec{d}):=\prod_{v_{s_{k}} \in V_{s}} p_{f}\left(v_{s_{k}}\right)^{d_{s_{k}}}\left(1-p_{f}\left(v_{s_{k}}\right)\right)^{1-d_{s_{k}}} .
$$

\section{Hidden Markov Model Hypotheses}

Let us denote the set of hypotheses as below:

$$
\begin{gathered}
H_{0}: \quad \text { no intruder activity presents } \\
H_{1}: \quad \text { an intruder activity presents } \\
\vdots \\
H_{M}: \quad M \text { intruder activities present }
\end{gathered}
$$

\footnotetext{
${ }^{2}$ For brevity of exposition, we assume that sensors are always operational and stationary. It is possible to extend the developed methodology of this paper to sensors with mobilities and/or sleep modes assuming that the locations and the modes of sensors are available for sensor data fusion. Also assumed is that a correct positive detection decision from a sensor occurs only if the intruder is co-located with the sensor. Relaxing this assumption is conceivable by considering the detection range of sensors (e.g., accounting the neighbor vertexes within the detection range) and assigning appropriate probabilities of detection and false alarm.
} 
Given the sensor models, we can build an HMM hypothesis for an intruder activity case as follows.

For $H_{p}, p \in\{1, \ldots, M\}$, we define a directed graph $G=$ $\left(V_{p}, E_{p}\right)$ where

$$
V_{p}:=\underbrace{V \times \ldots \times V}_{p} \text { and } E \subseteq V_{p} \times V_{p} .
$$

Let $v_{i}^{p}:=\left(v_{i_{1}}, \ldots, v_{i_{p}}\right) \in V_{p}$ and $v_{j}^{p}:=\left(v_{j_{1}}, \ldots, v_{j_{p}}\right) \in V_{p}$ where $v_{i_{k}} \in V$ and $v_{j_{k}} \in V$ represent the locations of the $k^{\text {th }}$ activity. Assuming statistical independence between the moving strategies of intruder activities, we have the state transition probability distribution for $H_{p}$ denoted by $A_{p}=$ $\left\{a_{v_{i}^{p}, v_{j}^{p}}\right\}$ where

$$
a_{v_{i}^{p}, v_{j}^{p}}:=p\left(X_{t+1}^{p}=v_{i}^{p} \mid X_{t}^{p}=v_{j}^{p}\right)=\prod_{k=1}^{p} a_{v_{i_{k}}, v_{j_{k}}} .
$$

The initial distribution is

$$
\pi_{1}\left(v_{i}^{p}\right)=\prod_{k=1}^{p} \pi_{1}\left(v_{i_{k}}\right) .
$$

The observation symbol probability distribution is $B=$ $\left\{b_{v_{j}^{p}}(\vec{d})\right\}$ where

$$
\begin{aligned}
b_{v_{j}^{p}}(\vec{d}) & :=p\left(D\left(V_{s}, t\right)=\vec{d} \mid X_{t}^{p}=v_{j}^{p}\right) \\
& =\prod_{k=1}^{n} p\left(d\left(v_{s_{k}}, t\right)=d_{s_{k}} \mid X_{t}^{p}=v_{j}^{p}\right) .
\end{aligned}
$$

For the above equation, if $\exists q \in\{1, \ldots, p\}$ s.t. $v_{j_{q}}=v_{s_{k}}$, $p\left(d\left(v_{s_{k}}, t\right)=d_{s_{k}} \mid X_{t}^{p}=v_{j}^{p}\right)=p_{d}\left(v_{s_{k}}\right)^{d_{s_{k}}}\left(1-p_{d}\left(v_{s_{k}}\right)\right)^{1-d_{s_{k}}}$.

Otherwise,

$p\left(d\left(v_{s_{k}}, t\right)=d_{s_{k}} \mid X_{t}^{p}=v_{j}^{p}\right)=p_{f}\left(v_{s_{k}}\right)^{d_{s_{k}}}\left(1-p_{f}\left(v_{s_{k}}\right)\right)^{1-d_{s_{k}}}$

In our model, we assumed that having multiple intruder activities at the same location does not increase the detection probability of the associated sensor. This can be defined differently. For instance, if sensors are likely to detect intruder activities better when there are more activities at the same location at the same time, then we may want to consider the following alternative approach: let $r$ be the number of $v_{s_{k}}$ components in $v_{j}^{p}=\left(v_{j_{1}}, v_{j_{2}}, \ldots, v_{j_{p}}\right)$. If $r \geq 1$, then

$$
p_{d}^{r}\left(v_{s_{k}}\right):=1-\left(1-p_{d}\left(v_{s_{k}}\right)\right)^{r} \text { and }
$$

$p\left(d\left(v_{s_{k}}, t\right)=d_{s_{k}} \mid X_{t}^{p}=v_{j}^{p}\right)=p_{d}^{r}\left(v_{s_{k}}\right)^{d_{s_{k}}}\left(1-p_{d}^{r}\left(v_{s_{k}}\right)\right)^{1-d_{s_{k}}}$

When $r=0$, we have

$p\left(d\left(v_{s_{k}}, t\right)=d_{s_{k}} \mid X_{t}^{p}=v_{j}^{p}\right)=p_{f}\left(v_{s_{k}}\right)^{d_{s_{k}}}\left(1-p_{f}\left(v_{s_{k}}\right)\right)^{1-d_{s_{k}}}$.

Let us recall the model in Fig. 2. To illustrate the above hypothesis construction procedure, we build $\mathrm{H}_{2}$ from the model in Fig. 2, which is depicted in Fig. 3. In Fig. 3, for the sake of readability, we drew only some part of the model. Darkened squares represent the pairs of intruder activity vertexes where sensors are deployed at least one of the vertexes. To illustrate, we compute the conditional

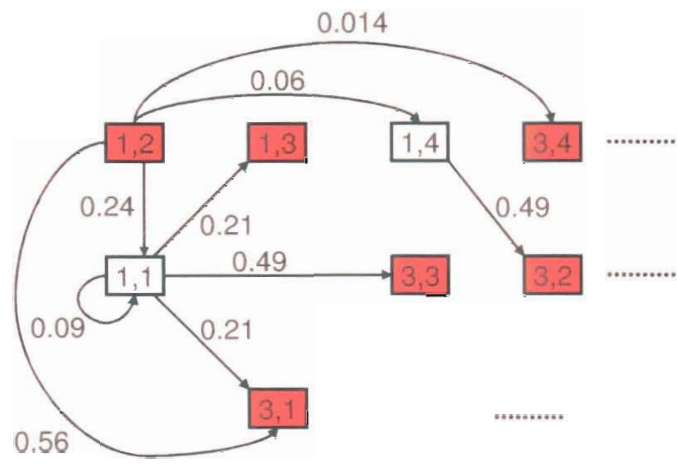

Fig. 3. Markov model of two intruder activities

observation probabilities when two intruder activities are present at $(1,2)$ pair below. Time index is omitted for brevity.

$$
\begin{aligned}
& p(D(\{2,3\})=(0,0) \mid(1,2))=\left(1-p_{d}(2)\right)\left(1-p_{f}(3)\right)=.24 \\
& p(D(\{2,3\})=(0,1) \mid(1,2))=\left(1-p_{d}(2)\right) p_{f}(3)=.06 \\
& p(D(\{2,3\})=(1,0) \mid(1,2))=p_{d}(2)\left(1-p_{f}(3)\right)=.56 \\
& p(D(\{2,3\})=(1,1) \mid(1,2))=p_{d}(2) p_{f}(3)=.14
\end{aligned}
$$

\section{Sequential Change Detection and ISOlation}

Having sequential data collected from sensors, we are interested in counting intruder activities in a sequential manner. Consider a sequence of independent random variables $Y_{1}, Y_{2}, \ldots$ Let $Y_{1}, Y_{2}, \ldots, Y_{v-1}$ be an i.i.d. sequence. Wald's Sequential Probability Ratio Test (SPRT) [21] has been used widely in testing which of two hypothesis probability density functions, say $f_{0}$ and $f_{1}$, better explains the sequential data. For two hypotheses to test, SPRT is an optimal sequential hypothesis test satisfying given probability detection and false alarm constraints.

Hypothesis testing approaches assume that the true hypothesis is given and does not change. When a sensor network is deployed initially, the domain to be monitored may have no on-going intruder activity. Later, intruders may enter to the domain to be monitored where the sensor network is deployed and operating. Therefore, intruder activity counting problem can be paraphrased into detecting the change of the true hypothesis from the hypothesis representing no intruder activity to some hypothesis representing some intruder activities.

Page's CUSUM algorithm [16] provides an optimal procedure for sequential change detection problem [10] between two hypotheses as SPRT for sequential hypothesis testing for i.i.d. sample sequences. Let us recall CUSUM algorithm. Consider a sequence of independent random variables $Y_{1}, Y_{2}, \ldots$ Let $Y_{1}, Y_{2}, \ldots, Y_{v-1}$ be i.i.d. sequence with probability density $f_{0}$, and $Y_{v}, Y_{v+1}, \ldots$ be i.i.d. sequence with probability density $f_{1}$. Time of change $v$ is not known.

Many sequential analysis techniques, including CUSUM, involve the following summation of log-likelihood ratio of 
probability densities:

$$
G_{j}^{i}:=\sum_{k=j}^{i} \log \left(\frac{f_{1}\left(Y_{k}\right)}{f_{0}\left(Y_{k}\right)}\right) .
$$

In particular, CUSUM involves finding the summation of loglikelihood ratio of probability densities that prefers density $f_{1}$ over $f_{0}$ most; i.e.,

$$
Q_{i}:=\max _{1 \leq j \leq i} G_{j}^{i} .
$$

Given a threshold $h>0$, the decision logic of CUSUM is simply to determine if

$$
Q_{i} \geq h
$$

The recursive form of this test is

$$
Q_{i}=\max \left(Q_{i-1}+\log \left(\frac{f_{1}\left(Y_{i}\right)}{f_{0}\left(Y_{i}\right)}\right), 0\right)
$$

with $Q_{0}=0$.

The stopping time of $N$ of CUSUM is the first time when $Q_{i} \geq h$. That is,

$$
N=\inf \left\{i \geq 1: Q_{i} \geq h\right\} .
$$

The stopping time $N$ is asymptotically optimal in the following sense [10]:

$$
E_{0}(N) \geq e^{h} \text { and } \bar{\tau}_{1}(N)=E_{1}(N) \sim \frac{h}{\rho} \text { as } h \rightarrow \infty .
$$

where $E_{0}^{\prime}(N)$ denotes the mean time before a false alarm, $\bar{\tau}_{1}(N)$ denotes the mean detection delay of a change, and $\rho$ is the Kullback-Leibler distance between the two probability densities, that is,

$$
\rho:=\int f_{1}(y) \cdot \log \left(\frac{f_{1}(y)}{f_{0}(y)}\right) d y .
$$

For multiple alternatives, consider a sequence of independent random variables $Y_{1}, Y_{2}, \ldots$ Let $Y_{1}, Y_{2}, \ldots, Y_{v-1}$ be i.i.d. sequence with probability density $f_{0}$, and $Y_{v}, Y_{v+1}, \ldots$ be i.i.d. sequence with probability density $f_{p} \in$ $\left\{f_{1}, \ldots, f_{M}\right\}$. Time of change $v$ to one of the alternative hypotheses is not known and we are interested in detecting and isolating the change from $f_{0}$ to $f_{p}$ in a sequential manner. Nikiforov's treatments in [13] generalize CUSUM procedure to accommodate multiple alternatives and show asymptotic optimality of the algorithm in minimizing the worst mean detection/isolation delay for a given mean time before a false alarm or a false isolation. A drawback of Nikiforov's procedure is that the proposed algorithm is not recursive. Subsequent developments in this line of research include [15], where Matrix-CUSUM provides a recursive procedure while preserving asymptotic optimality of Nikiforov's procedure.

Developments in $[13,15]$ only deal with i.i.d. sequences. Work presented in [2] deals with CUSUM procedure with the two HMM hypotheses where observation sequences are not independent. Combining the developments in [15] and [2], we develop our recursive algorithm for counting activities.
Before we proceed to present Matrix-CUSUM for multiple HMM hypotheses, we need the following preliminary computation. Consider the observation sequence $\left\{\vec{d}_{q}\right\}_{q=1}^{t}$ where

$$
\vec{d}_{q}:=\left(d_{q, 1}, d_{q, 2}, \ldots, d_{q, n}\right) \in\{0,1\}^{n} .
$$

The below shows a typical observation sequence with three sensors.

$$
\left(\begin{array}{l}
0 \\
0 \\
1 \\
\overrightarrow{d_{1}}
\end{array}\right)^{T} \rightarrow\left(\begin{array}{l}
0 \\
0 \\
0
\end{array}\right)^{T} \rightarrow \underset{d_{2}}{T} \rightarrow\left(\begin{array}{l}
0 \\
1 \\
0
\end{array}\right)^{T} \rightarrow \cdots
$$

Given the observation sequence $\left\{\vec{d}_{q}\right\}_{q=1}^{t}$, we are interested in computing the probability of observing $\left\{\vec{d}_{q}\right\}_{q=1}^{t}$ under HMM hypothesis $H_{p}$ denoted by $p\left(\left\{\vec{d}_{q}\right\}_{q=1}^{t} \mid H_{p}\right)$.

For the null hypothesis $H_{0}$,

$$
p\left(\left\{\vec{d}_{q}\right\}_{q=1}^{t} \mid H_{0}\right)=\prod_{1 \leq q \leq t, 1 \leq i \leq n} p_{f}\left(v_{i}\right)^{d_{q, i}}\left(1-p_{f}\left(v_{i}\right)\right)^{1-d_{q, i}} .
$$

For hypotheses other than $H_{0}$, we can use the forward algorithm [17] to compute conditional observation probability effectively. For the sake of completeness, we describe the forward algorithm in Appendix VIII-A.

Now we give our activity counting algorithm: Given $h>$ 0 , the pair $(\hat{N}, \hat{\delta})$ is defined by

$$
\hat{N}=\min \left\{\hat{N}^{1}, \hat{N}^{2}, \ldots, \hat{N}^{M}\right\} \text { and } \hat{p}=\arg \min _{1 \leq p \leq M} \hat{N}^{p}
$$

where

$$
\hat{N}^{p}=\inf \left\{i \geq 1: \min _{0 \leq k \neq p \leq M} \max _{1 \leq j \leq i} G_{j}^{i}(p, k) \geq h\right\}
$$

and

$$
G_{j}^{i}(p, k)=\log \left(\frac{p\left(\left\{\vec{d}_{q}\right\}_{q=j}^{i} \mid H_{p}\right)}{p\left(\left\{\vec{d}_{q}\right\}_{q=j}^{i} \mid H_{k}\right)}\right) .
$$

Note that CUSUM statistics of $p^{t h}$ hypothesis against $k^{\text {th }}$ hypothesis given by

$$
Q_{i}(p, k):=\max _{1 \leq j \leq i} G_{j}^{i}(p, k)
$$

is recursively computable. Therefore, the minimum CUSUM statistics of $p^{\text {th }}$ hypothesis against other hypotheses,

$$
\min _{0 \leq k \neq p \leq M} Q_{i}(p, k),
$$

is also recursively computable. The stopping time $\hat{N}$ is the instance when the minimum CUSUM statistics of one of hypotheses, say $H_{\hat{p}}$, becomes higher than the given threshold $h$. This is the moment when the procedure declares that the true hypothesis has changed from $H_{0}$ to $H_{\hat{p}}$. Obviously, the threshold $h$ affects the performance of the developed sequential decision process. For i.i.d. cases, roughly, with increasing $h$, expected time to reach the false detection and isolation decision increases exponentially while expected time to reach a correct detection and isolation decision increases linearly. See $[13,15]$ for more details. We conjecture that a similar argument should hold for our case as well. 


\section{TRACKing LOCATION AND EStimating Trace}

An estimate of the locations of $p$ activities at time $t$, denoted by $\hat{v}_{t}^{p}$, is computed as follows.

$$
\begin{gathered}
p\left(X_{t}^{p}=v_{i}^{p} \mid\left\{\vec{d}_{q}\right\}_{q=1}^{t}\right)=\frac{\alpha_{t}\left(v_{i}^{p}\right)}{p\left(\left\{\vec{d}_{q}\right\}_{q=1}^{t} \mid H_{p}\right)}, \\
\hat{v}_{t}^{p}:=\arg \max _{v_{i}^{p} \in V_{p}} p\left(X_{t}^{p}=v_{i}^{p} \mid\left\{\vec{d}_{q}\right\}_{q=1}^{t}\right) .
\end{gathered}
$$

Note that the forward algorithm in Appendix VIII-A computes the forward variable $\alpha_{t}\left(v_{i}^{p}\right)$ and the observation sequence probability $p\left(\left\{\vec{d}_{q}\right\}_{q=1}^{t} \mid H_{p}\right)$ recursively. Therefore, $\hat{v}_{t}^{p}$ can be recursively estimated on the fly.

Having the above Matrix-CUSUM procedure for multiple alternative HMM hypotheses, we use the following value as the estimate of the instance of hypothesis change:

$$
\hat{t}:=\arg \max _{1 \leq j \leq \hat{N}} G_{j}^{\hat{N}}(\hat{p}, 0)
$$

with 0 indicating that no intruder activity is present. Intuitively, $\hat{N}$ is the instance when Matrix-CUSUM for multiple alterative HMM hypotheses finalizes the isolation to the hypothesis $H_{\hat{p}}$. The value $\hat{t}$ means the beginning instance of observation subsequence that supports the change of hypothesis from $H_{0}$ to $H_{\hat{p}}$ best.

Given the estimate of the instance of hypothesis change, we are interested in finding the most probable trace of activities:

$$
\left\{\hat{v}_{t}^{\hat{p}}\right\}_{t=\hat{t}}^{\hat{N}}=\arg \max _{\left\{v_{t}^{\hat{p}}\right\}_{t=\hat{t}}^{\hat{N}}} p\left(\left\{X_{t}^{\hat{p}}\right\}_{t=\hat{t}}^{\hat{N}}=\left\{v_{t}^{\hat{p}}\right\}_{t=\hat{t}}^{\hat{N}} \mid\left\{\vec{d}_{q}\right\}_{q=\hat{t}}^{\hat{N}}, H_{\hat{p}}\right) .
$$

The Viterbi algorithm [20] finds the most probable sequence of hidden states that results in a sequence of observed events. For the sake of completeness, the Viterbi algorithm is described in Appendix VIII-B.

\section{ILLUSTRATIVE EXAMPLE}

Consider a graph $G=(V, E)$ depicted in Fig. 4. This

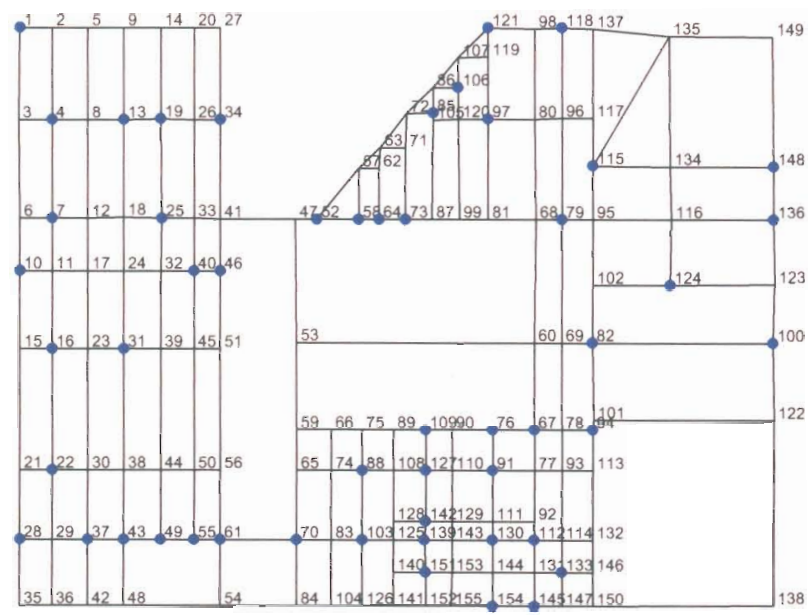

Fig. 4. Domain to be monitored graph $G$ includes 155 vertexes and 536 directed edges; all edges in Fig. 4 are bidirectional. That is,

$V=\{1,2, \ldots, 155\}$ and $E=\{(1,2),(2,1), \ldots,(154,155)\}$.

There are 52 stationary sensors (filled circles) in Fig. 4. Following the notation in the main presentation, we denote the sensor set by

$$
V_{s}:=\{3 i-2: i=1, \ldots, 52\}=\{1,4,7, \ldots, 154\} .
$$

Regarding false alarm and detection probabilities, for all $v \in V_{s}$, we assume that

$$
p_{f}(v)=0.01 \text { and } p_{d}(v)=0.95 \text {. }
$$

We assume that intruders enter to the network from any vertex and travel within the graph $G$ with independent Markov transition strategies. We also assume equally-likely entry probability among all vertexes. Therefore, for vertex $v \in V$,

$$
\pi_{1}(v)=\frac{1}{|V|}=\frac{1}{155} .
$$

For activity transition, we assume that, if $\left(v_{i}, v_{j}\right) \in E$,

$$
a_{v_{i}, v_{j}}=\frac{1}{\text { number of outgoing edges from } v_{i}} \text {; }
$$

i.e., all outgoing paths are equally probable. Otherwise, $a_{v_{i}, v_{j}}=0$. For example, $a_{1,2}=1 / 2$ and $a_{4,2}=1 / 4$.

We consider the following intruder activity hypotheses:

$$
\begin{array}{ll}
H_{0}: & \text { no activity presents } \\
H_{1}: & \text { single activity presents } \\
H_{2}: & 2 \text { activities present }
\end{array}
$$

Let us suppose that an activity enters the domain to be monitored at the $10^{\text {th }}$ sample step. The progress of the activity is depicted with the thick lines in Fig. 5(a); activity enters the monitored domain at vertex 1 and travels within the network following the thick lines. With this activity

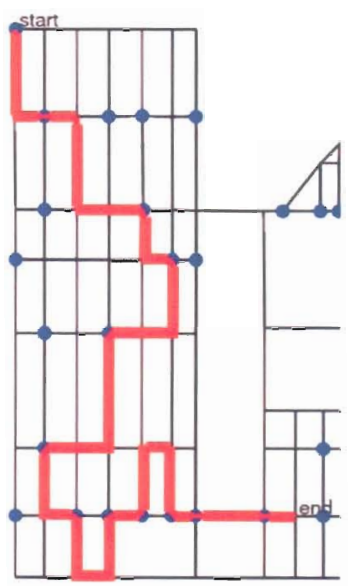

(a) Actual activity trace

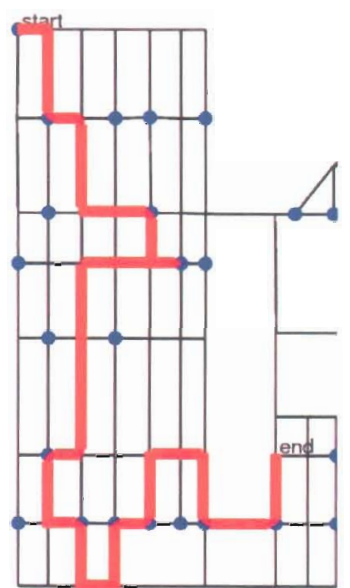

(b) The most probable activity trace
Fig. 5. Actual and inferred (via Viterbi algorithm) intruder activity traces 

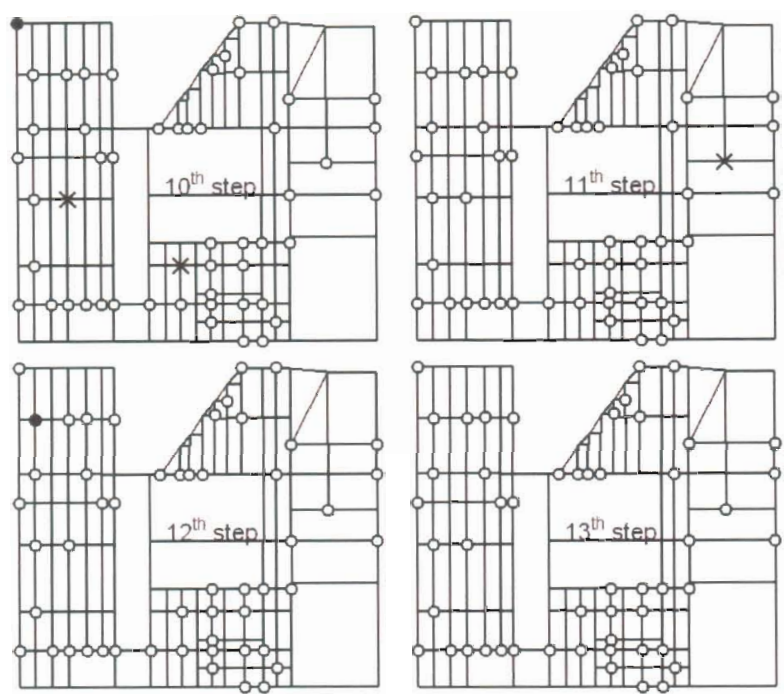

: correct activity detection

X : false alarm

Fig. 6. Observation snap shots

sample path, sequential observations are generated with the given false alarm and detection probabilities. Figure 6 gives the snapshots of the sequential observations from the steps 10 to 13 . Fquipped with the sequential observations of the

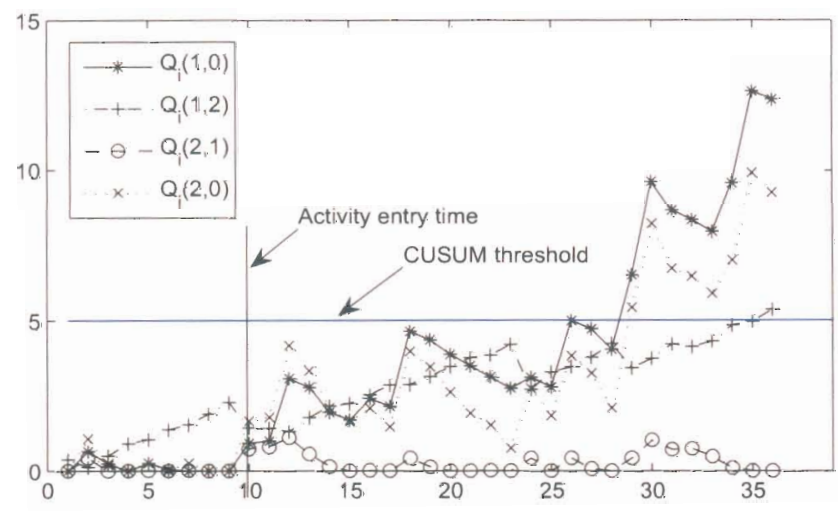

Fig. 7. Matrix-CUSUM progress

distributed sensors, we get the progress of Matrix-CUSUM depicted in Fig. 7. The threshold $h$ of Matrix-CUSUM is set to 5. The value $\left.\min \left(Q_{35}(1,0), Q_{35}(1,2)\right)\right)$ goes over 5 at the $36^{\text {th }}$ step while $\left.\min \left(Q_{35}(2,0), Q_{35}(2,1)\right)\right)$ stays below 5 ; this is the instance when Matrix-CUSUM for multiple alternative HMMs detects and isolates the change of true hypothesis from $H_{0}$ to $H_{1}$. With (1), we get the estimated activity entry time $\hat{t}=10$. Given this value, we run the Viterbi algorithm computing the most probable activity path, which is depicted in Fig. 5(b) with the thick lines.

\section{FINAL REMARKS}

The methodology presented in this paper is capable of detecting and isolating the change of the true hypothesis once. The problem of tracking the true hypothesis subsumes the problem addressed in this paper. We believe that the developed methodology should serve as a building block for hypothesis tracking problem, which is under current investigation.

The presented procedure of building model for multiple activities suffers with state space explosion as the number of activities increases. Compounded upon this, the forward and the Viterbi algorithms present square (of $\left|V_{p}\right|$ ) computational complexities per each recursion step. To handle largesize problems, our current research efforts also include the developments of algorithmic procedures and methodologies for alleviating this computational difficulty.

\section{ACKNOWLEDGEMENT}

The research reported in this paper was supported by the U.S. Department of Energy contract DE-AC07-05ID14517.

\section{REFERENCES}

[1] A. Arora, P. Dutta, S. Bapat, V. Kulathumani, H. Zhang, V. Naik, V. Mittal, H. Cao, M. Demirbas, M. Gouda, Y. Choi, T. Herman, S. Kulkarni, U. Arumugam, M. Nesterenko, A. Vora, and M. Miyashita. A line in the sand: a wireless sensor network for target detection, classification, and tracking. Comput. Networks, 46(5):605634, 2004.

[2] B. Chen and P. Willett. Detection of hidden Markov model transient signals. Aerospace and Electronic Systems, IEEE Transactions on, 36(4): 1253-1268, 2000.

[3] M. Chu, H. Haussecker, and F. Zhao. Scalable information-driven sensor querying and routing for ad hoc heterogeneous sensor networks. The International Journal of High Performance Computing Applications, 16(3):293-313, Fall 2002.

[4] T. Clouqueur, V. Phipatanasuphorn, P. Ramanathan, and K. K. Saluja. Sensor deployment strategy for target detection. In WSNA '02. Proceedings of the Ist ACM international workshop on Wireless sensor networks and applications, pages 42-48, New York, NY, USA, 2002. ACM Press.

[5] J. Corts, S. Martnez, T. Karatas, and F. Bullo. Coverage control for mobile sensing networks. IEEE Transactions on Robotics and Automation, 20(2):243-255, 2004.

[6] D. E. Denning. An intrusion-detection model. IEEE Trans. Softw. Eng., 13(2):222-232, 1987.

[7] H. Gonzlez-Banos. A randomized art-gallery algorithm for sensor placement. In SCG 2001: Proceedings of the 17th Annual Symposium on Computational Geometry, pages 232-240, New York, NY, USA, 2001. ACM Press.

[8] A. Howard, M. J. Matarić, and G. S. Sukhatme. Mobile sensor network deployment using potential fields: A distributed, scalable solution to the area coverage problem. In Proceedings of the International Symposium on Distributed Autonomous Robotic Systems, pages 299 308, 2002.

[9] D. Lee and A. Lin. Computational complexity of art gallery problems. IEEE Trans. Inf. Theor, 32(2):276-282, 1986.

[10] G. Lorden. Procedures for Reacting to a Change in Distribution. The Annals of Mathematical Statistics, 42(6):1897-1908, 1971.

[11] S. Meguerdichian, F. Koushanfar, M. Potkonjak, and M. B. Srivastava. Coverage problems in wireless ad-hoc sensor networks. In INFOCOM 2001: Proceedings of the 20th Annual Joint Conference of the IEEE Computer and Communications Societies, pages 1380-1387, 2001.

[12] S. Meguerdichian, F. Koushanfar, G. Qu, and M. Potkonjak. Exposure in wireless ad-hoc sensor networks. In MobiCom 2001: Proceedings of the 7 th anmual international conference on Mobile computing and networking, pages 139-150, New York, NY, USA, 2001. ACM Press.

[13] I. V. Nikiforov. A generalized change detection problem. IEEE Transactions on Information Theory, 41(1):171-187, 1995.

[14] J. O'Rourke. Art Gallery Theorems and Algorithms. Oxford University Press, 1987.

[15] T. Oskiper and H. V. Poor. Online activity detection in a multiuser environment using the matrix cusum algorithm. IEEE Transactions on Information Theory, 48(2):477-493, 2002. 
[16] E. S. Page. Continuous Inspection Schemes. Biometrika, 41(1/2):100115, 1954.

[17] L. R. Rabiner. A tutorial on hidden Markov models and selected applications inspeech recognition. Proceedings of the IEEE, 77(2):257-286, 1989.

[18] T. C. Shermer. Recent results in art galleries. Proc. of the IEEE, 80(9): $1384-1399,1992$.

[19] S. Tilak, N. B. Abu-Ghazaleh, and W. Heinzelman. A taxonomy of wireless micro-sensor network models. SIGMOBILE Mob. Comput. Commun. Rev, 6(2):28-36, 2002.

[20] A. Viterbi. Error bounds for convolutional codes and an asymptotically optimum decoding algorithm. Information Theory, IEEE Transactions on, $13(2): 260-269,1967$.

[21] A. Wald. Sequential Analysis. John Wiley \& Sons, 1947.

[22] T. Yoo and H. E. Garcia. Sensor deployment optimization for network intrusion detection. In Proceedings of 44th Annual Allerton Conference on Communication, Control, and Computing, pages $996-$ 1004, Urbana, IL, September 2006.

[23] Y. Zou and K. Chakrabarty. Sensor deployment and target localization in distributed sensor networks. Trans. on Embedded Computing Sys., 3(1):61-91, 2004.

\section{VIII, APPENDIX}

\section{A. Forward Algorithm}

Consider the forward variable

$$
\alpha_{t-1}\left(v_{i}^{p}\right)=p\left(\left\{\vec{d}_{q}\right\}_{q=1}^{t-1}, X_{t-1}^{p}=v_{i}^{p} \mid H_{p}\right) .
$$

The following recursion computes the forward variable $\alpha_{t}\left(v_{j}^{p}\right)$ effectively:

- Initial condition: for $v_{j}^{p} \in V_{p}$

$$
\alpha_{1}\left(v_{j}^{p}\right)=\pi_{1}\left(v_{j}^{p}\right) \cdot b_{v_{j}^{p}}\left(\vec{d}_{1}\right)
$$

- Induction: for $v_{j}^{p} \in V_{p}$

$$
\alpha_{t}\left(v_{j}^{p}\right)=\left[\sum_{v_{i}^{p} \in V_{p}} \alpha_{t-1}\left(v_{i}^{p}\right) \cdot a_{v_{j}^{p}, v_{i}^{p}}\right] \cdot b_{v_{j}^{p}}\left(\vec{d}_{t}\right)
$$

- Observation sequence probability:

$$
p\left(\left\{\vec{d}_{q}\right\}_{q=1}^{t} \mid H_{p}\right)=\sum_{v_{j}^{p} \in V_{p}} \alpha_{t}\left(v_{j}^{p}\right)
$$

The computational complexity of the forward algorithm of each recursion is $O\left(\left|V_{p}\right|^{2}\right)$ for $H_{p}$.

\section{B. Viterbi Algorithm}

Given the estimated entry time $\hat{t}$ of $\hat{p}$ activities, we can apply the Viterbi algorithm in order to compute the most probable activity path that best explains the sequence of observations from $\hat{t}$. The Viterbi algorithm is similar to the forward algorithm presented above. Instead of summing over transitions from incoming states, the associated recursion computes the maximum from incoming states as below. The term $\delta_{t}\left(v_{j}^{\hat{p}}\right)$ below implies the probability of the partial best path to $v_{j}^{p}$ at time $t$.

- Initial condition: for $v_{j}^{\hat{p}} \in V_{\hat{p}}$

$$
\delta_{\hat{t}}\left(v_{j}^{\hat{p}}\right)=\pi_{1}\left(v_{j}^{\hat{p}}\right) \cdot b_{v_{j}^{\hat{p}}}\left(\vec{d}_{\hat{t}}\right)
$$

- Induction: for $v_{j}^{\hat{p}} \in V_{\hat{p}}$

$$
\begin{gathered}
\delta_{t}\left(v_{j}^{\hat{p}}\right)=\left[\max _{v_{i}^{\hat{p}} \in V_{\hat{p}}} \delta_{t-1}\left(v_{i}^{\hat{p}}\right) \cdot a_{v_{j}^{\hat{p}}, v_{i}^{\hat{p}}}\right] \cdot b_{v_{j}^{\hat{p}}}\left(\vec{d}_{t}\right) \\
\psi_{t}\left(v_{j}^{\hat{p}}\right)=\left[\arg \max _{v_{i}^{\hat{p}} \in V_{\hat{p}}} \delta_{t-1}\left(v_{i}^{\hat{p}}\right) \cdot a_{v_{j}^{\hat{p}}, v_{i}^{\hat{p}}}\right]
\end{gathered}
$$

- Termination:

$$
\hat{v}_{\hat{N}}^{\hat{p}}=\arg \max _{v_{i}^{\hat{p}} \in V_{\hat{p}}} \delta_{\hat{N}}\left(v_{i}^{\hat{p}}\right)
$$

- Read out path:

$$
\hat{v}_{t}^{\hat{p}}=\psi_{t+1}\left(\hat{v}_{t+1}^{\hat{p}}\right), t=\hat{N}-1, \ldots, \hat{t} .
$$

The computational complexity of the Viterbi algorithm is $O\left(\left|V_{\hat{p}}\right|^{2} \cdot(\hat{N}-\hat{t})\right)$ for $H_{\hat{p}}$. 